\title{
Intracranial Hemangiopericytoma in a Child: A Case Report with Magnetic Resonance Spectroscopy Findings
}

\author{
Ali Murat KOC ${ }^{1}$, Ali Yusuf ONER ${ }^{1}$, Murat UCAR ${ }^{1}$, Alp Ozgun BORCEK ${ }^{2}$ \\ ${ }^{1}$ Gazi University, School of Medicine, Department of Radiology, Ankara, Turkey \\ ${ }^{2}$ Gazi University, School of Medicine, Department of Neurosurgery, Ankara, Turkey
}

\section{ABSTRACT}

Hemangiopericytoma is a hypervascular soft tissue sarcoma with a predilection for the extremities. It has two clinical types according to the age of presentation: Infantile hemangiopericytoma ( $<1$ years) and adult type hemangiopericytoma ( $>1$ years). The intracranial location is very rare and accounts for only $0.4-1 \%$ of all primary brain tumors, with only $10 \%$ detected in the pediatric age group. The differential diagnosis of this rare tumor from other extra-axial masses plays an important role in proper treatment planning. There are few case reports in adult patients indicating that magnetic resonance spectroscopy (MRS) can play a role in the differential diagnosis. Here, we present imaging findings of this rare tumor, including MRS at 3.0T of an intracranial hemangiopericytoma in a 4.5 year old boy.

KEYWORDS: Hemangiopericytoma, MR Spectroscopy, Intracranial, Childhood

\section{INTRODUCTION}

Hemangiopericytoma (HPC) is a hypervascular soft tissue sarcoma of the body, arising from pericytes of Zimmerman (1, 4). Although it can be seen anywhere where capillary vessels are found, the most common site for HPC is the lower limbs followed by the head and neck region, retroperitoneum and visceral organs $(4,7)$. Central nervous system involvement is rare and accounts for only $0.4-1 \%$ of all primary brain tumors $(1,2)$. It is classically an adult type of tumor with only $10 \%$ of cases seen in the pediatric group (2). Imaging plays an important role in the differentiation of HPC from other extraaxial mass lesions and proper treatment planning. Conventional computed tomography (CT) and magnetic resonance imaging (MRI) findings of this rare tumor are well defined, with few case reports $(1,6)$ in the literature pointing to a possible role of magnetic resonance spectroscopy (MRS) in the adult population. However, to the best of our knowledge, there is no report discussing MRS features of HPC in the pediatric age group. In this paper we present imaging findings, including MRS at 3.0T, of an infant with intracranial HPC.

\section{CASE REPORT}

A 4.5 year-old boy, suffering from seizure, nausea and vomiting symptoms, was referred to our hospital. His symptoms were present from birth, with progression in the last 3 months. At the time of his hospitalization, he had no neurological deficit but experienced seizures of the generalized tonic clonic type with normal laboratory findings. Unenhanced cranial computerized tomography (CT) showed a mass at the left frontal lobe containing calcifications and cystic areas (Figure 1). MRI performed on a 3T scanner revealed an extra-axial mass abutting the falx showing prominent enhancement, compressing the adjacent brain parenchyma with accompanying edema, and causing midline shift (Figure 2A,B). Multivoxel MRS (TR/TE/NEX: 1700 / 35 and 135 $\mathrm{msec} / 3)$ placed over the frontal lesion showed a prominent choline and a shallow glutamate peak with absent N-Acetyl Aspartate, pointing to a mass of non-glial origin (Figure $3 \mathrm{~A}$, $B)$. The patient underwent surgery with total excision of the mass, which turned out to be an HPC on pathology. The boy is under follow-up with no seizures and tumor relapse at 18 months after surgery. 


\section{DISCUSSION}

HPC is a hypervascular soft tissue sarcoma. Although initially named as "angioblastic meningioma", it has been realized in the following years that this is not a meningioma (1). In the last classification of World Health Organization (WHO), HPC is included in the "extrapleural HPC and solitary fibrous tumor" subgroup which is under the group of "fibroblastic / myofibroblastic tumors" (5). Furthermore, intracranial HPC is divided into two categories: WHO Grade III Anaplastic Hemangiopericytoma and WHO Grade II Hemangiopericytoma $(2,6)$. HPC has two clinical types according to the age of presentation; infantile hemangiopericytoma ( $<1$ years) and

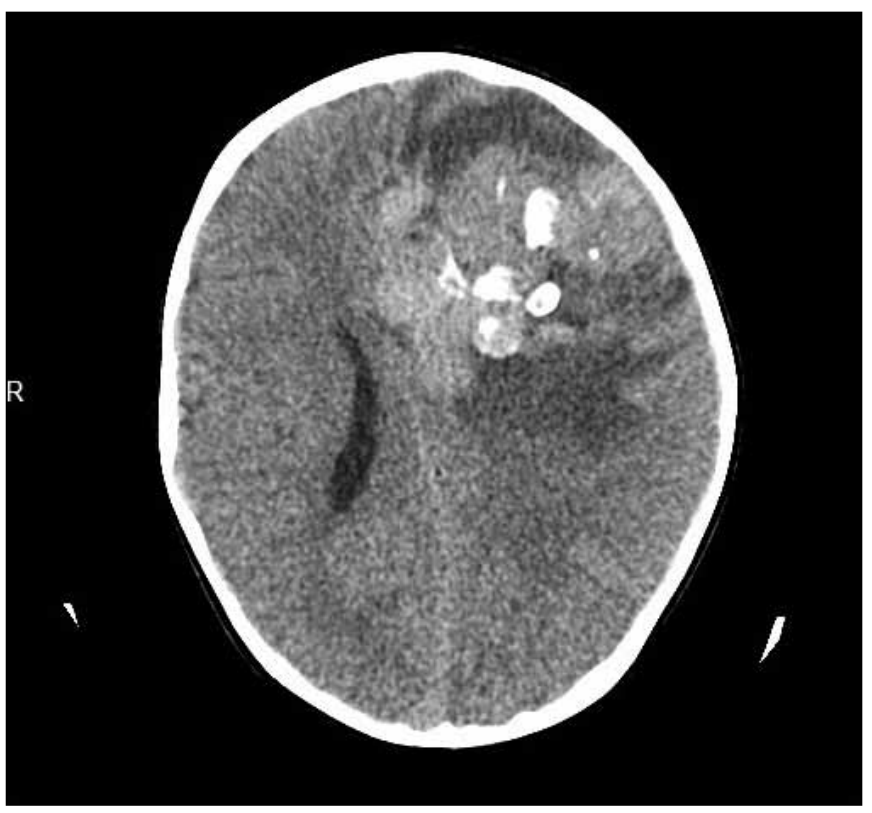

Figure 1: Non-enhanced axial cranial CT reveals an extra-axial mass lesion on the left frontal region with prominent calcifications. adult type hemangiopericytoma ( $>1$ years), with the second type being more common $(4,7)$.

Intracranial HPC and meningiomas have similar symptoms and similar appearances. However, the clinical courses and treatment methodologies are different. First of all, HPCs are more aggressive tumors. They can locally recur and can metastasize to distant organs $(1,7)$. Treatment of HPCs and meningiomas involve surgery with the aim of complete resection $(4,7)$. Because HPCs are highly vascularized, it is important to diagnose them preoperatively to avoid loss of high volumes of blood during surgery. Preoperative embolisation of the tumor can also be considered to minimize anticipated blood loss $(1,7)$. Postoperative radio- or chemotherapy is usually administered in HPCs whereas surgery alone is adequate in meningiomas $(1,7)$. Because infantile type hemangiopericytomas are less aggressive than adult types (6), their response to chemotherapy is better (7), and surgery may be adequate by itself.

HPC and meningiomas are both extra-axial tumors presenting with similar imaging findings, including dural tail and enhancement after contrast injection. However, there are some clues, although not fully discriminative, that may help in differentiating HPCs from meningiomas. HPCs have more heterogenous texture, enhancement pattern and narrower dural tail $(2,6)$. Calcifications usually point to meningiomas instead of HPCs, with some rare exceptions. To the best of our knowledge, the above reported patient is the seventh case of HPC presenting with calcifications $(2,6)$.

MRS is known to complement conventional MRI and increase diagnostic accuracy in the imaging workup of different intracranial tumors. In the recent literature, there are few reports concerning the use of MRS in HPC patients. Barba et al. (1) evaluated MRS findings in three adult patients with HPC at 1.5T. They report absence of NAA, and moderate peaks of Choline and Glutamate together with prominent myoinositol in those patients and conclude that the higher contribution of
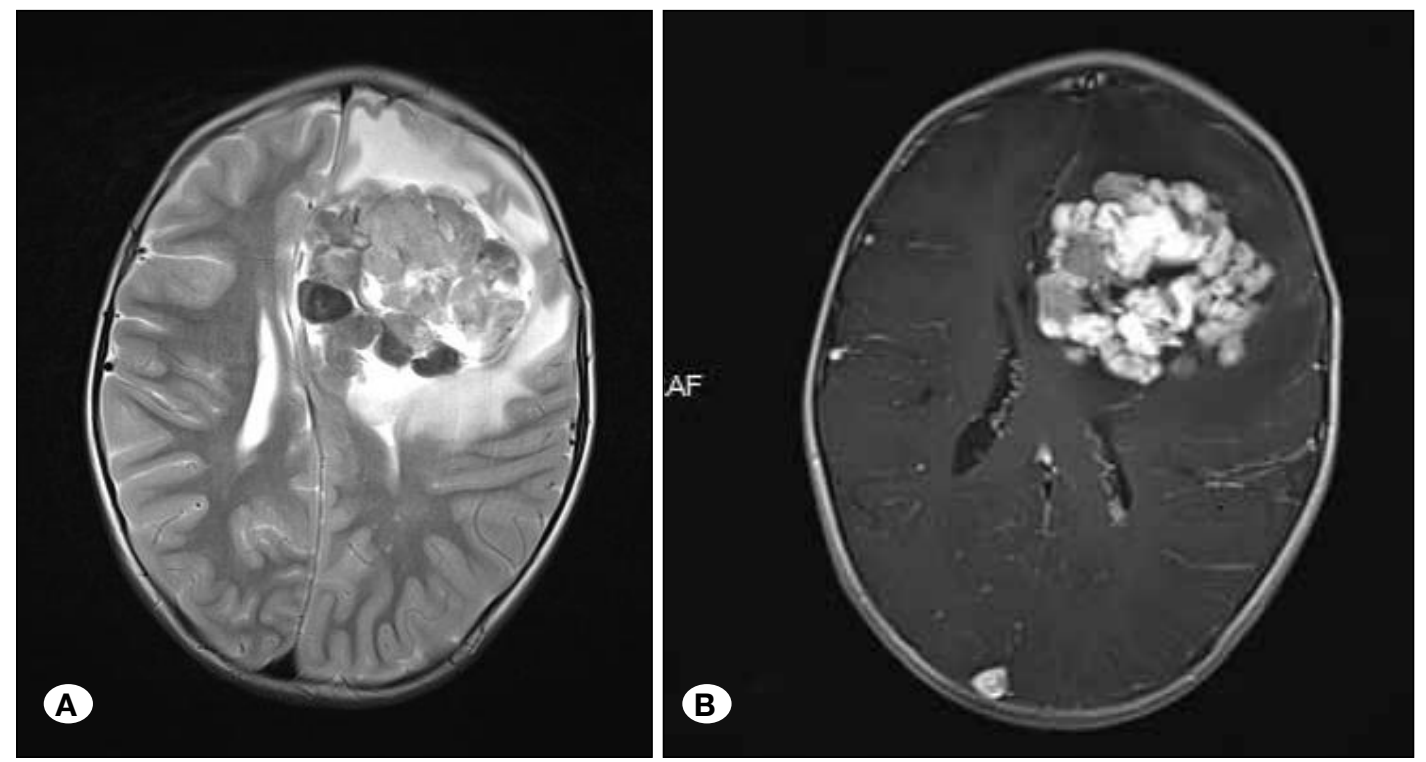

Figure 2: Axial T2weighted $(\mathbf{A})$ and postcontrast T1-weighted (B) MRI images reveal a mass with heterogenous signal intensity and heterogenous contrast enhancement. Peripheral edema and a midline shift are also noted. 

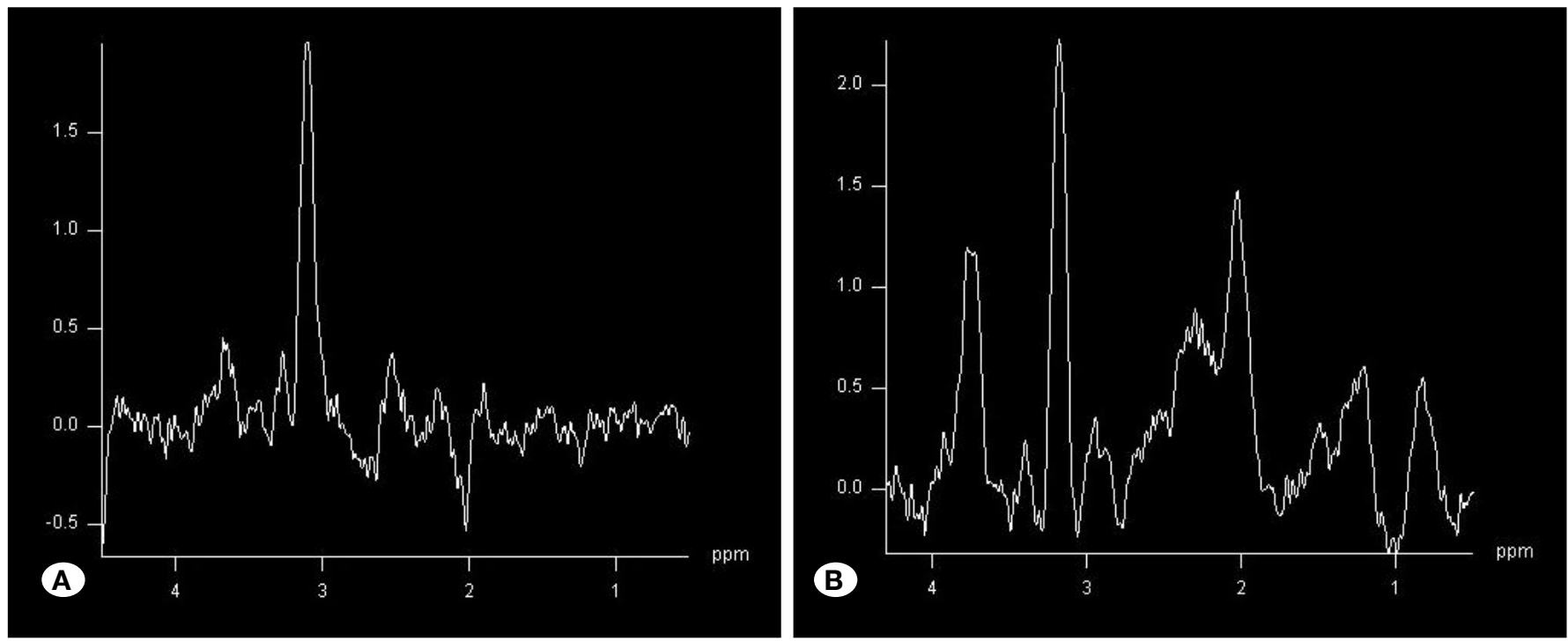

Figure 3: Multivoxel MRS performed at the same level as in figure 2 with two different echo times. Intermediate echo (135 msec) MRS shows the absence of $\mathrm{N}$-Acetyl Aspartate, a prominent choline peak at $3.2 \mathrm{ppm}$ with a shallow glutamate peak around $2.35 \mathrm{ppm}$ (A). A repeated MRS with short echo time $(30 \mathrm{msec})$ at the same level documents myoinositol to be within the normal range $(\mathbf{B})$.

myoinositol than glycine at the $3.56 \mathrm{ppm}$ peak can be used as an important discriminator of HPC. They also found that trimethylamine (choline) levels of hemangiopericytomas are higher than that of meningiomas. N-Acetyl Aspartate (NAA) is the neuronal marker of the normal brain parenchyma, and therefore is absent in any extra-axial mass (8). Choline is the second largest peak of the normal brain and receives contribution from glycerophosphocholine, phosphocholine and phosphatidylcholine. It is an established marker of cellular membrane turnover reflecting cellular proliferation, as expected in any neoplastic process (8). Glutamate (Glu) and Glutamine (GIn) are usually found together as an inseparable complex at 2.05-2.50 ppm (8). Glu is an important excitatory neurotransmitter and plays a role in energy metabolism. Metabolic alterations under the form of absent NAA, and presence of glutamate and choline as detected for the above presented case are in strong agreement with neuropathological changes known to occur in HPC. However, these MRS findings are nonspecific and can accompany any extraaxial mass. On the other hand, short and moderate TE MRS of the abovementioned pediatric case failed to depict the peak of myoinositol reported in adult HPC patients $(1,6)$. Myoinositol is thought to be the most important non-nitrogenous osmolyte in the mammalian brain and has been identified as a glialspecific marker. Myoinositol is in fact at its peak in neonates (3), but decreases rapidly and reaches its final value by the end of the first year of life. After this period, its increase is associated with gliosis, astrocytosis and Alzheimer's disease or myelin breakdown (8). The reported presence of myoinositol in adult HPC patients most probably reflects glial proliferation and gliosis that accompany this tumor with a relatively slow proliferation rate. Considering the history of seizures from birth for the above presented pediatric patient, the absence of myoinositol can be explained by the hypothesis that the tumor was present since the prenatal stage and that the high plasticity of the brain during neonatal and infantile periods prevented the occurrence of gliosis.

To conclude, imaging workup of HPCs in the pediatric age can fail to show any MRS findings that might help their differentiation from meningiomas, in contrast to the adult type. Radiological differentiation of these rare hypervascular tumors may therefore be more difficult than in the adult population.

\section{- REFERENCES}

1. Barba I, Moreno A, Martinez-Perez I, Tate AR, Cabanas ME, Baquero M, Capdevila A, Arus C: Magnetic resonance spectroscopy of brain hemangiopericytomas: High myoinositol concentrations and discrimination from meningiomas. J Neurosurg 94:55-60, 2001

2. Chen $Q$, Chen $X Z$, Wang JM, Li SW, Jiang T, Dai JP: Intracranial meningeal hemangiopericytomas in children and adolescents: CT and MR imaging findings. Am J Neuroradiol 33:195-199, 2012

3. Dezortova M, Hajek M: $1 \mathrm{H}$ MR spectroscopy in pediatrics. European Journal of Radiology 67:240-249, 2008

4. Fernandez-Pineda I, Parida L, Jenkins JJ, Davidoff AM, Rao BN, Rodriguez-Galindo C: Childhood hemangiopericytoma: Review of St Jude Children's Research Hospital. J Pediatr Hematol Oncol 33:356-359, 2011

5. Fletcher CDM: The evolving classification of soft tissue tumours: An update based on the new WHO classification. Histopathology 48:3-12, 2006

6. Kalra V, Fenn K, Vortmeyer A, Malhotra A: Intracranial infantile hemangiopericytoma: A distinctive and more benign clinical entity. Neurographics 2:119-125, 2012

7. Marec-Berard P: (January 2003, update April 2004) Malignant hemangiopericytoma. Orphanet Encyclopedia https://www. orpha.net/data/patho/GB/uk-HPC.pdf Accessed 19 April 2014

8. Soares DP, Law M: Magnetic resonance spectroscopy of the brain: Review of metabolites and clinical applications. Clinical Radiology 64:12-21, 2009 